ESJ Humanities

\title{
Contraintes d'acces aux Soins de Sante Face a la Croissance Demographique de la Zone Sanitaire Ouidah/Kpomasse/Tori-Bossito
}

\author{
Tchinkoun Ayathe Carine \\ Azonhe Hervé Thierry \\ Ecole Doctorale Pluridisciplinaire "Espace, culture et Santé" \\ Département de Géographie et Aménagement du Territoire, \\ Université d'Abomey-Calavi, Bénin \\ Dako Kpacha Sabine $M$. \\ Université de Parakou, \\ Laboratoire des Climatologie et Ethnoclimatologie Tropicales, Bénin \\ Hounkponou Prince Edouard \\ Ecole Doctorale Pluridisciplinaire "Espace, culture et Santé" \\ Département de Géographie et Aménagement du Territoire, \\ Université d'Abomey-Calavi, Bénin
}

Doi:10.19044/esj.2021.v17n36p183

Submitted: 28 September 2021

Accepted: 14 October 2021

Published: 31 October 2021
Copyright 2021 Author(s)

Under Creative Commons BY-NC-ND

4.0 OPEN ACCESS

Cite As:

Tchinkoun A.C., Azonhe H.T., Dako Kpacha S.M. \& Hounkponou P.E. (2021). Contraintes d'acces aux Soins de Sante Face a la Croissance Demographique de la Zone Sanitaire Ouidah/Kpomasse/Tori-Bossito. European Scientific Journal, ESJ, 17 (36), 183.

https://doi.org/10.19044/esj.2021.v17n36p183

\section{Résumé}

La nécessité, voire l'exigence de disposer des services de santé de qualité, accessibles géographiquement s'impose à une population sans cesse en croissance. Les infrastructures sanitaires, les équipements médicaux, la disponibilité du personnel soignant nécessaire pour accueillir et prendre en charge cette population font toujours défaut. La présente étude aborde les contraintes d'accès aux soins de santé face à l'explosion démographique de la zone sanitaire Ouidah/Kpomassè/Tori-Bossito. Les observations, la recherche documentaire et les travaux de terrain sont les principales étapes de la méthodologie adoptée. Les données utilisées ont été collectées auprès de 900 ménages et 78 personnes ressources. L'Indice de Confort Matériel (ICM) a été calculé pour déterminer le confort des enquêtés. Les Analyse Factorielle des 
Composantes (AFC) ont été réalisées pour évaluer le lien entre l'accès aux soins de santé et les différentes variables indépendantes. Le modèle SWOT (Strenght, Weaknesses, Opportunities, Threats) a permis d'évaluer les contraintes liées à l'accessibilité aux soins face à la croissance démographique observée dans la zone sanitaire Ouidah/Kpomassè/Tori-Bossito (OKT). Les résultats ont révélé que la disponibilité, la répartition des infrastructures sanitaires et du personnel soignant, le confort matériel du ménage, l'état de praticabilité des voies d'accès aux centres de santé conditionnent l'accessibilité aux soins des ménages. Malgré les efforts fournis par l'Etat et les collectivités territoriales, l'offre de soins de santé est insuffisante au regard du croît démographique.

Mots-clés : Ouidah/Kpomassè/Tori-Bossito, croissance démographique, contraintes, accès, soins de santé

\title{
Constraints of Access to Health Care Facing the Demographic Growth of the Ouidah/Kpomasse/Tori-Bossito Health Zone
}

\author{
Tchinkoun Ayathe Carine \\ Azonhe Hervé Thierry
}

Ecole Doctorale Pluridisciplinaire "Espace, culture et Santé"

Département de Géographie et Aménagement du Territoire,

Université d'Abomey-Calavi, Bénin

Dako Kpacha Sabine M.

Université de Parakou,

Laboratoire des Climatologie et Ethnoclimatologie Tropicales, Bénin

\section{Hounkponou Prince Edouard}

Ecole Doctorale Pluridisciplinaire "Espace, culture et Santé"

Département de Géographie et Aménagement du Territoire,

Université d'Abomey-Calavi, Bénin

\section{Abstract}

The need, indeed the requirement, to have quality health services that are geographically accessible is imposed on an ever-growing population. Health infrastructure, medical equipment and the availability of the nursing staff necessary to welcome and take care of this population are still lacking. This study addresses the constraints of access to healthcare in the face of the demographic explosion in the Ouidah/Kpomassè/Tori-Bossito health zone. Observations, documentary research and fieldwork are the main stages of the methodology adopted. The data used was collected from 900 households and 78 resource persons. The Material Comfort Index (ICM) was calculated to 
determine the comfort of the respondents. The Component Factor Analysis (AFC) was carried out to assess the link between access to health care and the various independent variables. The SWOT model (Strength, Weaknesses, Opportunities, Threats) made it possible to assess the constraints related to access to care in the face of the demographic growth observed in the Ouidah/Kpomassè/Tori-Bossito (OKT) health zone. The results revealed that the availability and distribution of health infrastructure and nursing staff, the material comfort of the household, the state of practicability of access routes to health centers condition access to household care. Despite the efforts made by the State and local authorities, the supply of health care is insufficient in view of the demographic growth.

Keywords: Ouidah / Kpomassè / Tori-Bossito, population growth, constraints, access, health care

\section{Introduction}

La zone sanitaire Ouidah/Kpomassè/Tori-Bossito (OKT) à l'instar des zones sanitaires du Bénin a connu une croissance démographique au cours des dernières décennies avec pour corollaire l'essoufflement du système public de santé qui n'arrive pas à s'adapter au rythme démographique (SANTANA, 2016). La population de la zone est passée de 151659 habitants en 1992 à 331689 habitants en 2018 (INSAE, 2013). Cette croissance démographique exerce une très grande influence sur l'évolution de la demande sociale notamment en matière de services de santé (PNDS, 2009). Le Bénin à l'instar des pays en développement, se trouve alors dans une situation délicate : la couverture sanitaire, mesurée en termes de disponibilité d'infrastructures, de densité ou de desserte médicales ou encore de lits d'hospitalisation, y est limitée (Richard, 2001). Malgré les efforts fournis par l'Etat et les autorités locales, l'offre de soins de santé n'arrive toujours pas à suivre la demande de la population de la zone OKT en matière de santé. Ainsi, une grande partie de la population se sent exclue, ou s'exclue du système de santé pour plusieurs raisons: accessibilité géographique, insuffisance de personnels soignants qualifiés, incapacité financière, etc.

Dans ce contexte où la croissance démographique a une influence inéluctable sur l'organisation et la structuration du système de santé du fait d'un déficit en ressources humaines et matérielles, il est impérieux d'étudier les contraintes actuelles à l'accessibilité aux soins de santé des communautés.

La présente recherche aborde donc la question des difficultés d'accès aux soins de santé dans un contexte d'accroissement démographique dans la zone sanitaire Ouidah/Kpomassè/Tori-Bossito. Elle présente la situation sanitaire actuelle de la zone d'étude, les gaps selon les normes de l'OMS et les contraintes à lever pour améliorer la situation. 
La Zone sanitaire Ouidah/Kpomassè/Tori-Bossito est située au Sud du département de l'Atlantique entre $6^{\circ} 17^{\prime} 25^{\prime \prime}$ et $6^{\circ} 37^{\prime} 27$ de latitude Nord et entre 1 ${ }^{\circ} 56^{\prime} 13^{\prime \prime}$ ' et $2^{\circ} 16^{\prime} 29$ de longitude Est (Bureau de zone OKT, 2020). Elle est limitée au Nord par la commune d'Allada, à l'Est par la zone sanitaire d'Abomey-Calavi et la commune de Zê, à l'Ouest par le lac Ahémé qui constitue une frontière naturelle avec le département du Mono, au Sud par l'Océan Atlantique sur une distance d'environ $60 \mathrm{~km}$. La figure 1 présente les situations géographique et administrative de la zone sanitaire OKT.

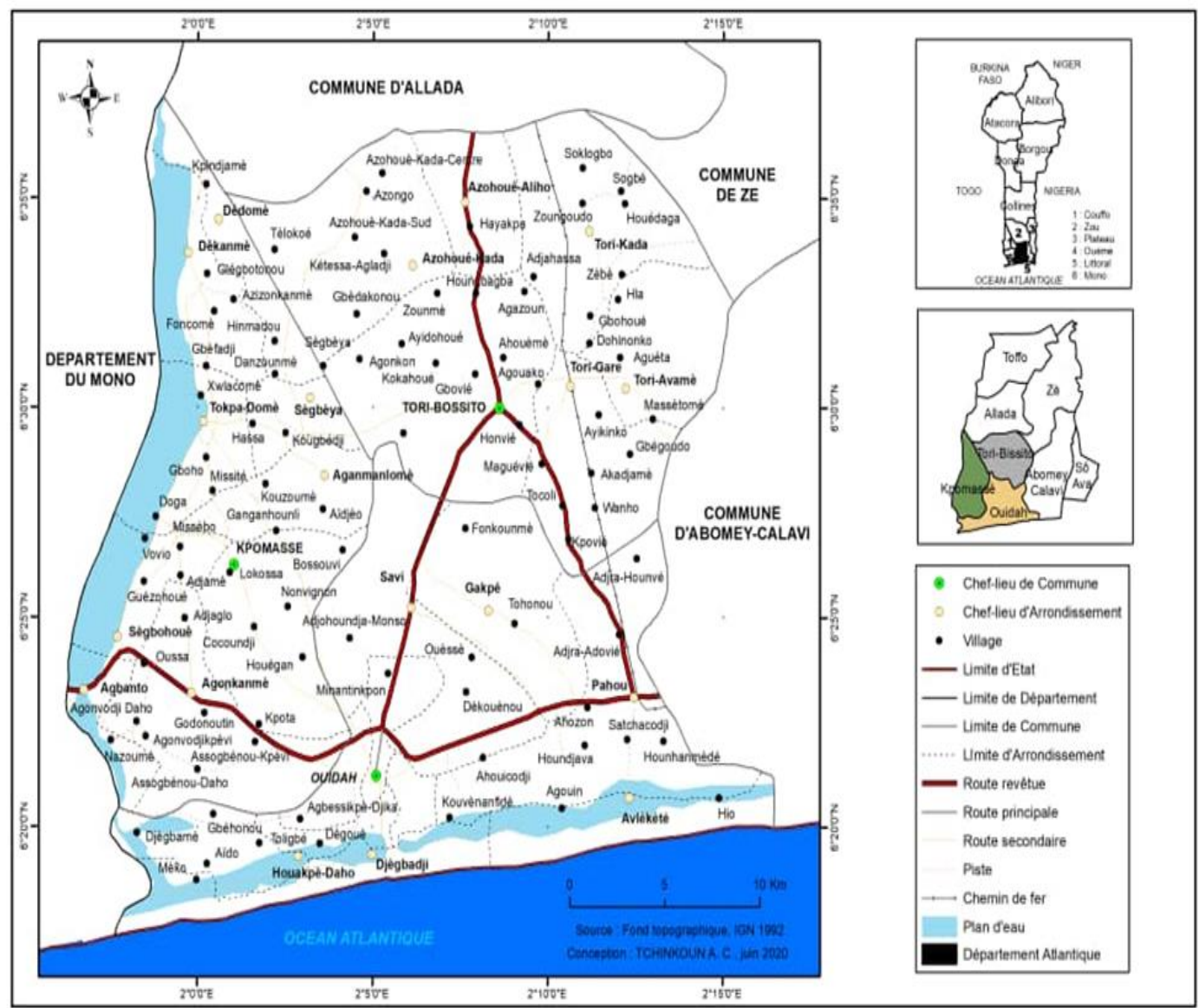

Figure 1 : Situations géographique et administrative de la zone sanitaire OKT

\section{Donnees et Methodes}

\subsection{Données collectées}

Pour étudier les difficultés d'accès aux soins de santé dans la zone OKT, des données quantitatives et qualitatives provenant de diverses sources ont été collectées. Il s'agit des données démographiques issues des recensements de la population de 1992, 2002 et 2013, de la répartition des 
infrastructures sanitaires, de la disponibilité du personnel de santé, de la capacité financière des ménages.

\subsection{Méthodes}

\subsubsection{Collecte des données}

Le premier groupe cible est constitué des ménages des communes de Ouidah, Kpomassè et Tori-Bossito. A ces ménages s'ajoutent les autorités communales, les élus locaux et les responsables des différentes formations sanitaires publiques et privées de la zone sanitaire.

\section{Matériels et outils de collecte des données}

Les matériels utilisés pour la collecte des données sont: un GPS pour relever les coordonnées géographiques des différentes formations sanitaires, un appareil photographique pour la prise des vues illustratives, l'application ODK Collect installée sur des tablettes pour la collecte des données socioéconomiques, un carnet de note et des porte-fiches. Trois outils d'investigation ont été utilisés. Il s'agit : d'un questionnaire à l'endroit des chefs de ménages, des guides pour les entretiens avec les agents de santé, les responsables des centres de santé communaux des trois communes de la zone sanitaire, les élus locaux des trois communes de la zone sanitaire et d'une grille d'observation.

Les données ont été collectées à travers les trois axes que sont : la recherche documentaire, les enquêtes puis les observations.

\section{Echantillonnage et échantillon}

Compte tenu de la population d'étude, deux méthodes d'échantillonnage ont été retenues. Il s'agit de la méthode probabiliste appliquée aux groupes de ménages et la méthode non probabiliste qui a pris en compte les personnes ressources auprès de qui il a été collecté des informations relatives à la situation sanitaire de la zone OKT face à la croissance démographique.

Tous les arrondissements de la zone d'étude ont été impliqués dans la présente étude et leur échantillon représentatif est déterminé. Le nombre de ménage enquêtés a donc été défini par la méthode de SCHWARTZ (1995). Sa formule se présente comme suit :

Avec :

$$
\mathrm{N}=\frac{Z \alpha^{2} P q}{d^{2} x F C}
$$

$\mathrm{N}$ : taille de l'échantillon

$\mathrm{Z} \alpha$ : Niveau de confiance déduit du taux de confiance (1.96 pour $95 \%$ )

$\mathrm{P}$ : taux d'accroissement de la population de 2002 à 2013

$\mathrm{q}: 1-\mathrm{P}$ 
$\mathrm{d}:$ marge d'erreur égale à $5 \%$

$\mathrm{Fc}$ : facteur de correction de l'effet grappe ici égal à 4

La taille de l'échantillon dans chaque commune est récapitulée dans le tableau I.

Tableau I : Répartition par commune de l'échantillon

\begin{tabular}{|c|c|c|}
\hline Communes & Effectif des ménages & Ménages à enquêter \\
\hline OUIDAH & 36459 & 405 \\
\hline KPOMASSE & 15280 & 270 \\
\hline TORI-BOSSITO & 11879 & 225 \\
\hline OKT & $\mathbf{6 3 6 1 8}$ & $\mathbf{9 0 0}$ \\
\hline
\end{tabular}

Source : Résultats d'enquêtes et calculs, Janvier 2021

\subsubsection{Traitement des données et analyse de résultats}

La base de données obtenue a été apurée et les données exportées dans le logiciel SPSS, version 25.0 pour la tabulation. La tabulation a été réalisée grâce au même logiciel. La mise en forme des tableaux et graphiques a été faite avec les logiciels Excel 2013 et Word 2013. Une fois que la base de données est traitée, il a été procédé à des analyses.

Des analyses bi variée et multi variées sont faites pour comprendre les associations et les forces des relations entre la variable dépendante qu'est « l'accès aux soins de santé » et les variables indépendantes que sont «l'ICM », «le coût des services, des actes en santé », "l'effet de la distance sur l'utilisation des services de soins de santé », « la répartition des infrastructures sanitaires modernes publiques et privées», « la disponibilité de personnels soignants », « le niveau d'instruction des interrogés » et «l'état des voies d'accès ». Ces analyses sont faites pour apprécier la ou les variable (s) indépendante(s) qui influencent surtout la variable dépendante.

La zone tampon ou le buffer est une méthode d'analyse spatiale qui consiste à créer une zone d'extension aux dimensions définies (appelées rayon) par l'opérateur, autour d'un point, d'une ligne ou d'un polygone. Ainsi, des zones tampons de cinq (05) kilomètres de rayon ont été fixées autour des formations sanitaires publiques pour mettre en évidence les liens de proximité entre les formations sanitaires publiques et les populations. Des zones tampons d'un (01) kilomètre de rayon ont également été fixées autour des formations sanitaires publiques en estimant que cette distance est suffisamment petite pour être parcourue à pied avec les jeunes enfants, les vieilles personnes et en peu de temps. 


\section{Resultats et Discussion}

L'offre de soins de santé en quantité et en qualité, continue d'être un casse-tête pour les pouvoirs publics béninois qui sont confrontés aux défis de la pression démographique. Cette situation est aggravée avec le contexte démographique actuel.

\subsection{Caractéristiques de la population de la zone sanitaire OKT}

Les caractéristiques démographiques de la zone sanitaire OKT peuvent être appréhendées grâce aux indicateurs de valeurs absolus (taille de la population, densité) analysés à travers le tableau II.

Tableau II : Evolution de la population et des densités par commune

\begin{tabular}{|c|r|r|r|r|r|r|r|r|r|}
\hline Communes & $\begin{array}{c}\text { Sup au } \\
\mathrm{km}^{2}\end{array}$ & $\begin{array}{c}\text { Populati } \\
\text { on en } \\
1992\end{array}$ & $\begin{array}{r}\text { Densité } \\
\text { en 1992 }\end{array}$ & $\begin{array}{c}\text { Populati } \\
\text { on en } \\
2002\end{array}$ & $\begin{array}{r}\text { Densité } \\
\text { en 2002 }\end{array}$ & $\begin{array}{c}\text { Populati } \\
\text { on en } \\
2013\end{array}$ & $\begin{array}{r}\text { Densité } \\
\text { en 2013 }\end{array}$ & $\begin{array}{c}\text { Populati } \\
\text { on } \\
\text { estimée } \\
\text { en 2018 }\end{array}$ & $\begin{array}{c}\text { Densité } \\
\text { estimée } \\
\text { en 2018 }\end{array}$ \\
Ouidah & 337 & 64433 & 192 & 76555 & 228 & 162034 & 481 & 187064 & 555 \\
\hline Kpomassè & 294 & 50059 & 170 & 57190 & 194 & 67648 & 230 & 78098 & 266 \\
\hline $\begin{array}{c}\text { Tori- } \\
\text { Bossito }\end{array}$ & 329 & 37167 & 113 & 44569 & 136 & 57632 & 175 & 66535 & 202 \\
\hline OKT & 960 & 151659 & 159 & 178314 & 186 & 287314 & 296 & 331697 & 341 \\
\hline
\end{tabular}

Les résultats des Recensements Généraux de la population et de l'habitat RGPH (1992 à 2013) et la projection en 2018 faite par l'INSAE (Institut National de la Statistique et de l'Analyse Economique) montrent que la population de la zone sanitaire OKT a augmenté de façon significative passant de 151659 habitants en 1992 à 331697 habitants en 2018, soit un coefficient multiplicateur de 2,19. La densité de population de la zone OKT quant à elle, est passée de $159 \mathrm{hab} / \mathrm{km}^{2}$ en 1979 à $341 \mathrm{hab} / \mathrm{km}^{2}$. Elle est en forte croissante dans toutes les communes à ces différentes dates. Cette pression de la croissance démographique est appréhendée dans la zone d'étude à partir des contraintes sur l'offre des services de soins de santé.

\subsection{Couverture en infrastructures sanitaires et normes selon l'OMS dans la zone OKT}

Bien que le taux de couverture en infrastructures sanitaires soit acceptable pour l'ensemble de la zone, l'offre publique de services de santé est encore insuffisante, au regard de la taille de la population. Les normes OMS en matière d'infrastructures sanitaires sont d'un hôpital de zone pour 150.000 habitants, un centre de santé de commune pour 50.000 habitants et un centre de santé d'Arrondissement pour 10.000 habitants (OMS citée par BONI (2014)). Le tableau 3 présente la disponibilité des infrastructures sanitaires publiques de la zone OKT et les normes selon l'OMS. 
Tableau III : Normes OMS et Infrastructures sanitaires publiques de la zone OKT

\begin{tabular}{|c|c|c|c|c|c|c|c|c|}
\hline & $\begin{array}{l}\text { Pop totale } \\
\text { en } 2018\end{array}$ & $\begin{array}{l}\mathrm{HZ} \\
\text { disponible }\end{array}$ & $\begin{array}{l}\text { HZ/norm } \\
\text { es de } \\
\text { l'OMS }\end{array}$ & $\begin{array}{l}\text { CSC } \\
\text { disponible }\end{array}$ & $\begin{array}{l}\text { CSC/nor } \\
\text { mes de } \\
\text { l'OMS }\end{array}$ & $\begin{array}{l}\text { CSA } \\
\text { disponible }\end{array}$ & $\begin{array}{l}\text { CSA/ } \\
\text { normes de } \\
\text { l'OMS }\end{array}$ & $\begin{array}{l}\text { Besoins en } \\
\text { infrastruc } \\
\text { tures }\end{array}$ \\
\hline Kpomassè & 78098 & & 1 & 1 & 2 & 9 & 8 & $1 \mathrm{CSC}$ \\
\hline Ouidah & 187064 & & 1 & 1 & 4 & 14 & 19 & $\begin{array}{l}3 \mathrm{CSC}, 5 \\
\mathrm{CSA}\end{array}$ \\
\hline $\begin{array}{l}\text { Tori- } \\
\text { Bossito }\end{array}$ & 66535 & & 0 & 1 & 2 & 8 & 7 & $1 \mathrm{CSC}$ \\
\hline $\begin{array}{l}\text { Total ZS } \\
\text { OKT }\end{array}$ & 331697 & 1 & 2 & 3 & 8 & 31 & 36 & $\begin{array}{l}\text { 1HZ, } \\
\text { 5CSC, } \\
5 \mathrm{CSA}\end{array}$ \\
\hline
\end{tabular}

Source : Résultats d'enquêtes et calculs, Janvier 2021

$H Z$ : Hôpital de zone ; CSC : Centre de Santé Communal ; CSA : Centre de Santé d'Arrondissement

De l'analyse des résultats du tableau III, il ressort que des efforts ont été fournis par l'Etat et les collectivités locales dans la construction des infrastructures sanitaires dans la zone sanitaire OKT. Sur les 36 CSA et 8 CSC nécessaires selon les normes de l'OMS, 31 CSA et 3 CSC sont en activité. Il ne reste donc que 10 centres de santé à construire sur 45 soit une couverture d'infrastructure sanitaire de $77,77 \%$. Selon les normes de l'OMS, la zone sanitaire OKT devrait avoir deux (2) hôpitaux de zone mais elle n'en a qu'un seul.

La réorganisation du système sanitaire béninois intervenu en 1997, prévoit une zone sanitaire pour une population allant de 100.000 à 200.000 habitants. La zone sanitaire compte aujourd'hui environ 331.697 habitants mais reste toujours avec une seule zone sanitaire.

Il a été remarqué au cours des enquêtes que peu d'infrastructures disponibles sont conformes aux normes de l'OMS surtout dans la Commune de Ouidah où les centres de santé sont vétustes, et ne disposent pas de logement pour le personnel soignant. Il faut noter qu'au cours de la période de 2012 à 2017, le plateau technique de la zone sanitaire a connu une amélioration grâce au Financement Basé sur les Résultats (FBR).

Les arrondissements de Ouidah IV et d'Agbanto n'ont pas encore de centre de santé d'arrondissement alors que le nombre d'habitants dans ces localités est sans cesse croissant. D'après le dernier recensement général de la population et de l'habitation (RGPH4) de 2013, les arrondissements de Ouidah IV et Agbanto comptaient respectivement 9475 et 5694 habitants (INSAE, 2013). C'est le centre de santé privé " Adolph kolping"' qui fait office de centre de santé d'arrondissement dans l'arrondissement de Agbanto. L'absence de centre de santé dans l'arrondissement de Ouidah IV rend les populations plus vulnérables à toutes sortes de maladies surtout pour rallier un centre de santé de niveau périphérique. 
La figure 2 présente la répartition des infrastructures sanitaires dans la zone sanitaire OKT.

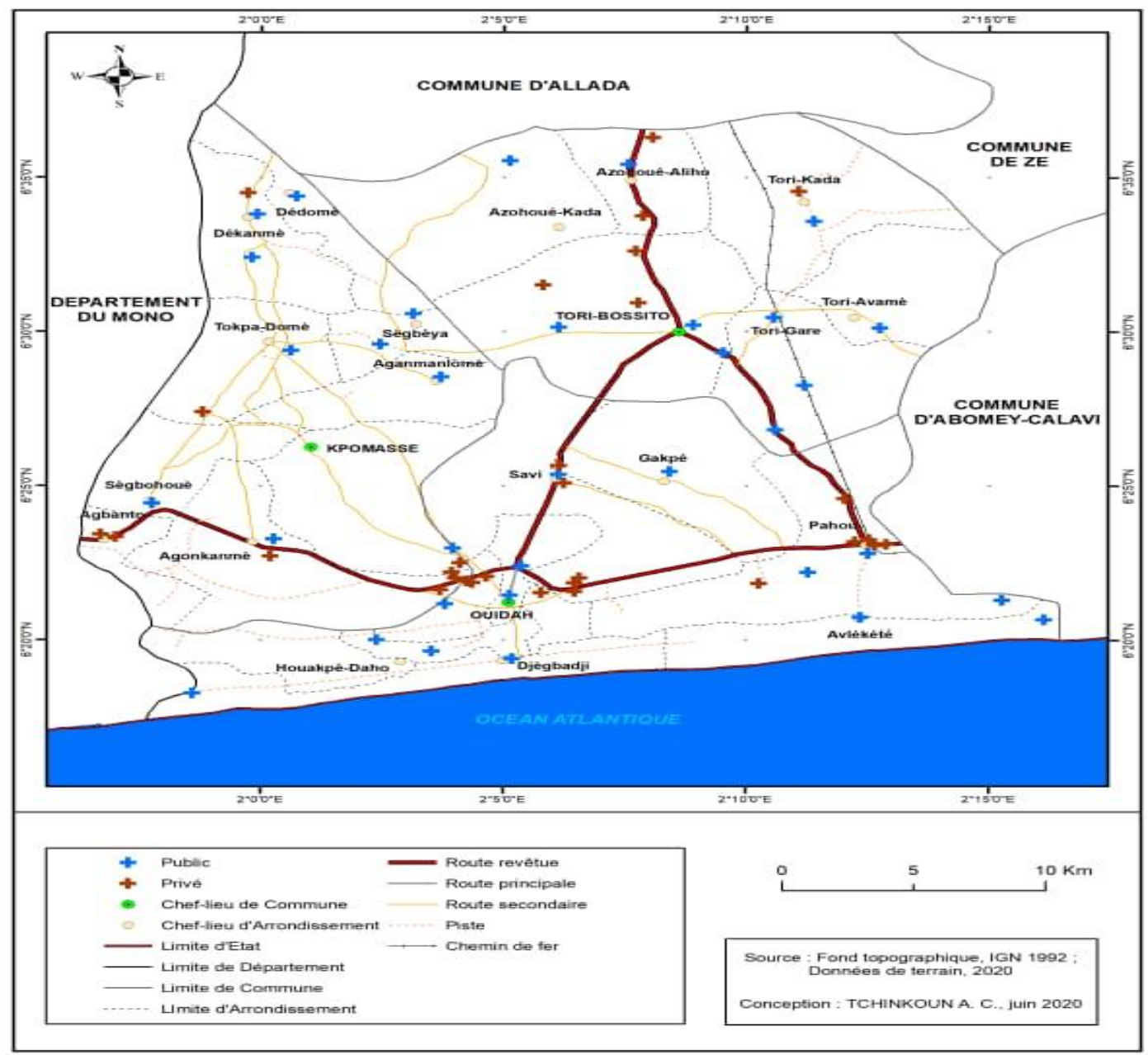

Figure 2: Répartition des infrastructures sanitaires dans la zone OKT

De la figure 2, on retient que la zone sanitaire dispose de trente-cinq (35) centres de santé publics fonctionnels, de deux (02) centres de détection de la tuberculose, d'un (01) centre de détection de la lèpre, de trente-cinq (35) centres de santé privés autorisés et de deux (02) centres confessionnels. La commune de Kpomassè dispose de dix (10) centres de santé publics fonctionnels, de trois centres de santé publics non fonctionnels et de quatre (04) centres de santé privés autorisés. La commune de Ouidah dispose quant à elle de seize (16) centres de santé publics fonctionnels, d'un centre de santé public non fonctionnels, d'un centre de détection de la tuberculose, d'un centre de détection de la lèpre, d'un centre de santé confessionnel et de vingt-six (27) 
centres de santé privés autorisés. Celle de Tori-Bossito compte neuf (9) centres de santé publics tous fonctionnels et cinq (5) centres de santé privés autorisés. On remarque une inégale répartition spatiale des centres de santé dans la zone d'étude. Cela s'explique par le fait que pour des raisons politiques, certaines autorités ou élus locaux exercent une influence pour dévier dans leur localité l'installation des infrastructures de santé.

\subsection{Personnel soignant}

De nombreux spécialistes sont déployés dans les formations sanitaires de la zone OKT pour répondre à la demande en soins de santé des populations. En se référant à la taille de la population, on pourrait s'interroger sur l'effectif du personnel disponible et les réels besoins sanitaires de ces populations. Pour mesurer la relation entre la densité de la population et l'effectif du personnel soignant disponible, les ratios médecin/habitants, IDE/habitants et celui $\mathrm{SDE} /$ habitants sont calculés en tenant compte des normes de l'OMS qui sont de 1 Médecin pour 10000 habitants, 1 IDE pour 5000 habitants et 1 SFE pour 5000 habitants (OMS citée par BONI (2014)). La figure 3 présente la comparaison du personnel de santé disponible aux normes de l'OMS par commune.

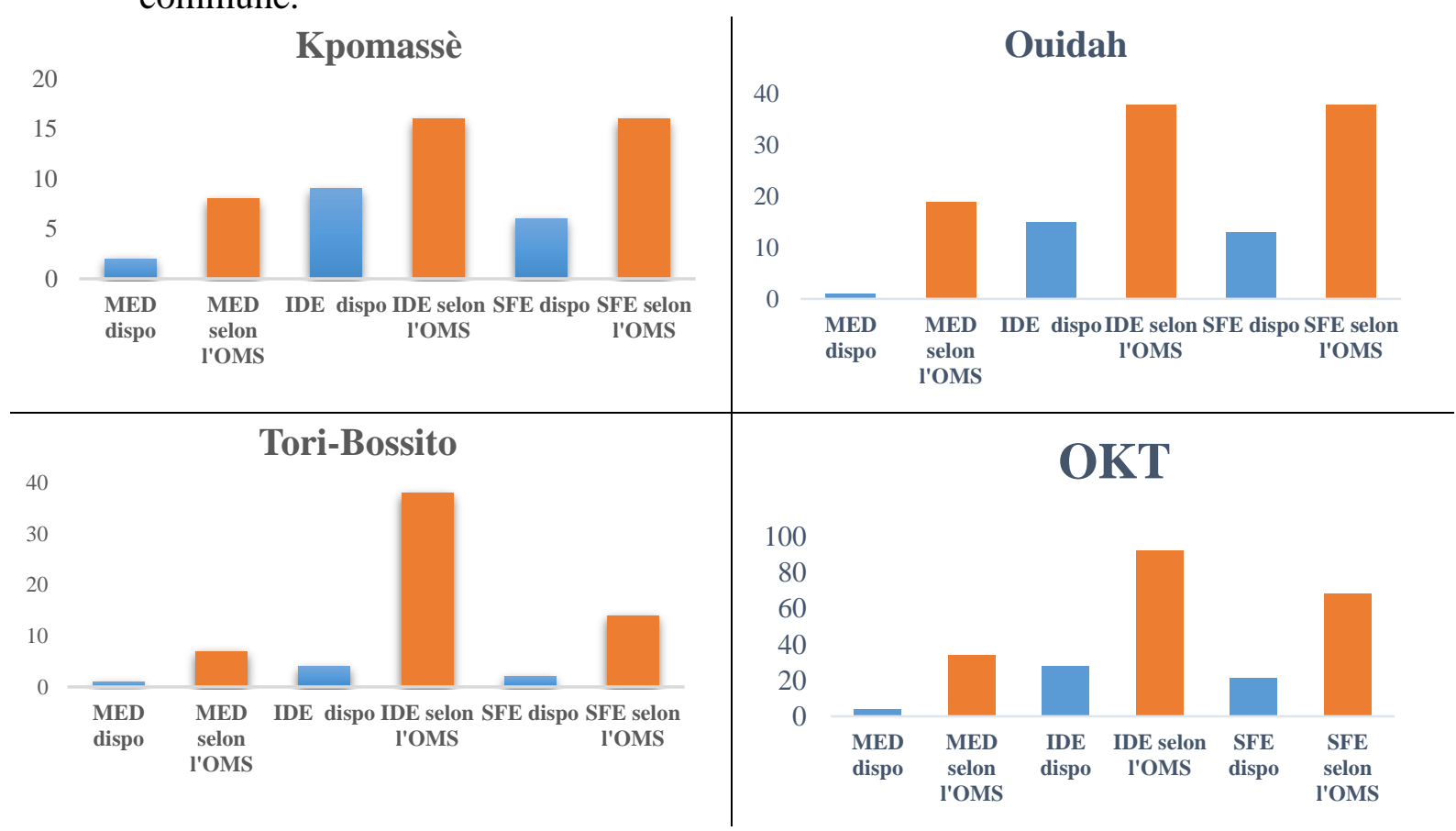

Figure 3 : Comparaison du personnel de santé disponible aux normes de l'OMS par commune et dans la zone sanitaire OKT

Source : Résultats d'enquêtes et calculs, Janvier 2021

IDE : Infirmier Diplômé d'Etat ; SFE : Sage-femme d'Etat ; MED : Médecin 
L'analyse de la figure 3 montre qu'il y a quelques disparités entre les aires sanitaires. En effet, le ratio de 1 médecin pour 10.000 habitants n'est atteint dans aucune des communes. La zone sanitaire OKT compte trois (04) médecins sur les trente-quatre (35) qu'il en faut.

Il existe un grand écart entre l'effectif de médecin disponible en rapport avec la taille de la population. Un recrutement de trente et un (30) médecins s'impose pour toute la zone sanitaire. Le même phénomène est observé au niveau des Infirmiers Diplômés d'Etat (IDE). Sur les quatre-vingtdouze (92) infirmiers diplômés d'Etat dont la zone sanitaire a besoin pour répondre aux attentes sanitaires de la population selon les normes de l'OMS, elle n'en compte que vingt-huit (28). Un équilibre s'impose alors avec une mutation de cinquante-quatre (54) IDE.

Les centres de santé tels que : Houakpè-Daho, Ganlononcodji, Adounko, Houndjava, Djègbadji, Djondji, Akadjamè, Kpassè, Ouidah2, Aganmalomè, Agonkanmey, Dékanmè, Avamè, Azohouè-Aliho, AzohouèCada, Acadjamè, Houénonko, Hèkandji n'ont pas d'IDE. Certains centres disposent d'au moins 01 IDE mais aucun ne respecte les normes de l'OMS en rapport avec le personnel IDE disponible. Un recrutement de soixante-quatre (64) IDE s'impose donc à l'Etat et aux collectivités locales afin de respecter les normes de l'OMS.

L'effectif des sages-femmes d'Etat (SFE) dans tous les CSA est inférieur à la norme de l'OMS. Aucun des CSA ne respecte les normes de l'OMS en rapport avec le personnel SFE disponible. Un recrutement de quarante-sept (47) SFE est nécessaire pour répondre aux besoins actuels des populations.

On retient que le personnel soignant est insuffisant malgré les efforts fournis par l'Etat pour recruter du personnel dans les centres de santé de la zone OKT. Certains centres de santé ne disposent pas d'infirmiers diplômés d'Etat et de sages-femmes et tout repose souvent sur les aides-soignantes et/ou infirmières brevetées qui ne sont pas habiletés à administrer des soins. La preuve que le problème est beaucoup plus au niveau du personnel soignant que des infrastructures sanitaires. Toutefois, chacun des centres souffre du manque soit de salle d'hospitalisation, de laboratoire, de boîtes à pansement, de lits, de tensiomètre, de tables d'accouchement, ou d'obus d'oxygène, etc.

\subsection{Contraintes géographiques de l'accès aux soins de santé dans la zone sanitaire OKT}

La friction de la distance a un impact sur le recours aux soins de santé. Or atteindre un centre de santé suppose qu'il faut franchir une distance, un certain nombre d'obstacles lié au relief, aux voies d'accès, etc. 


\section{Répartition des usagers selon l'éloignement du premier centre de santé}

Plus un centre de santé est proche, plus vite on peut s'y rendre et plus il est loin, plus on a des difficultés pour y aller. La figure 4 présente la répartition des usagers selon l'éloignement du premier centre de santé.

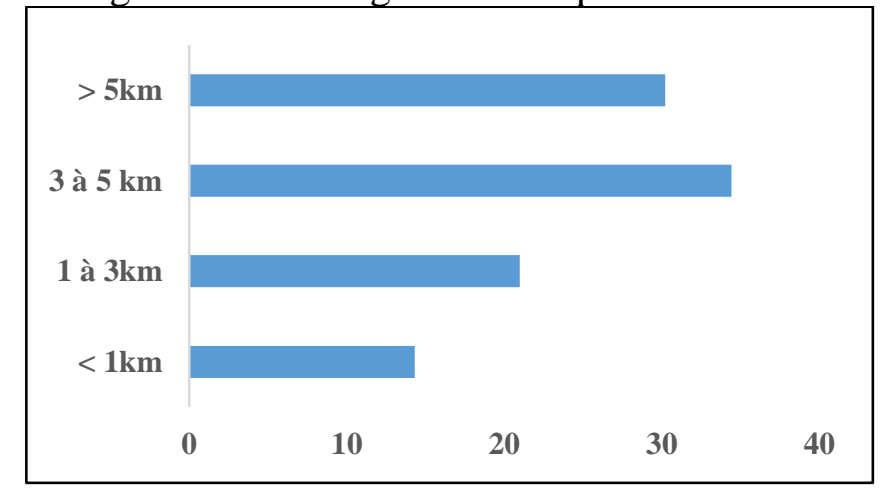

Figure 4 : Répartition des usagers selon l'éloignement du premier centre de santé dans la zone OKT

Source : Résultats d'enquêtes de terrain, février 2021

On retient de l'analyse de la figure 4 que $30,22 \%$ des ménages se trouvent à plus de 5 kilomètres du premier centre de santé. L'éloignement des centres de santé publics oblige les malades soit à rester chez eux et pratiquer l'automédication, soit à s'intéresser aux guérisseurs traditionnels ou à aller vers des centres de santé privés qui sont moins éloignés. Dans le même sens, $48,3 \%$ des personnes enquêtées estiment qu'il leur faut trente (30) minutes pour se rendre dans le centre de santé qu'ils fréquentent à pieds. Ceci pose un véritable problème d'accessibilité géographique aux centres de santé publics.

\section{Méthode des zones tampons}

Le maillage géographique des centres de santé publics justifie le temps de parcours pour s'y rendre. Selon l'OMS, le premier centre de santé doit être au plus à $5 \mathrm{~km}$ des populations (OMS citée par BONI (2014)). Les zones tampons de $5 \mathrm{~km}$ de rayon autour des formations sanitaires publiques dans la zone sanitaire OKT sont présentées par la figure 5. 


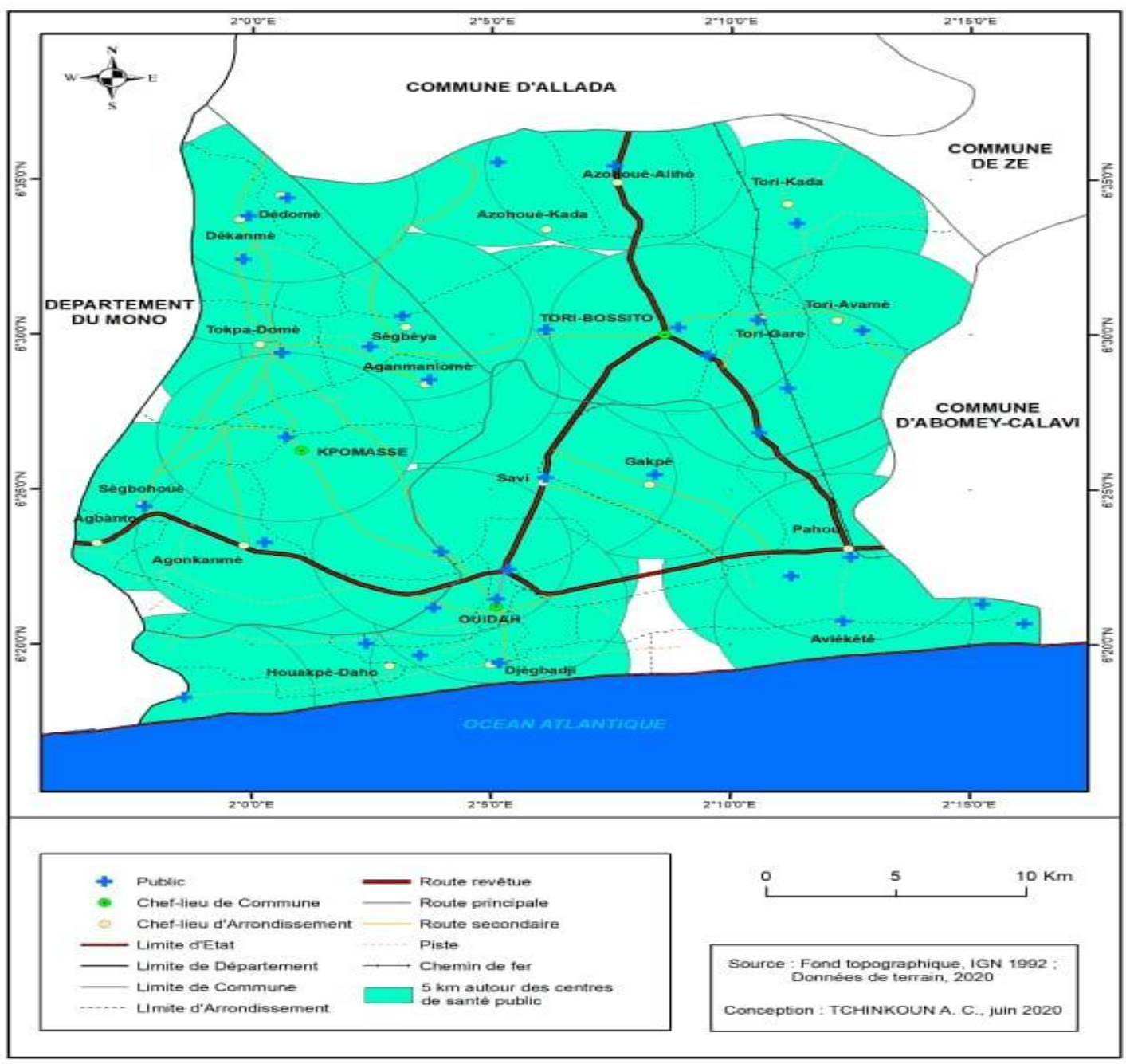

Figure 5 : Zones tampons de $5 \mathrm{~km}$ de rayon autour des formations sanitaires publiques dans la zone sanitaire OKT

Il ressort de l'analyse de la figure 5 , que les zones tampons de $5 \mathrm{~km}$ définies autour des formations sanitaires publiques se chevauchent largement. Ce maillage montre que la couverture sanitaire de la zone OKT est dense et donc les formations sanitaires publiques peuvent être ralliées facilement par les populations. Toutefois quelques déserts sanitaires ou espaces de ségrégation sanitaire sont observés à : Lokossa dans l'arrondissement de Aganmalomè, à Vovio dans l'arrondissement de Sègbohouè, à Doga et Missèbo dans l'arrondissement de Kpomassè centre, à Nazoumè dans l'arrondissement de Agbanto et à Ahouicodji et Adjra-Hounvè dans l'arrondissement de Pahou. La grande superficie de la zone sanitaire (ZS) OKT $\left(960 \mathrm{~km}^{2}\right)$ est à la base de ces poches de déserts sanitaires. 


\section{Etat de praticabilité des voies}

La fréquentation des centres de santé dépend surtout de la praticabilité des voies d'accès. Certains phénomènes rendent parfois difficile le parcours pour atteindre les centres de santé. Les pistes et voies non bitumées sont pour la plupart très dégradées dans la zone sanitaire du fait surtout de l'inondation alors qu'elles sont les plus pratiquées par la population pour aller dans leur centre de santé. En effet les voies d'accès aux centres de santé dans la zone sanitaire sont pour la plupart impraticable et ce en toute saison du fait des sols, des pentes et surtout des précipitations. Seules les voies menant vers les centres de santé de Ganlononcodji, Kpassè, Kpoviè, Savi, Kindji, Kouénonko, Azohouè Aliho, Tori-Bossito, Sègbohouè, Adolphkolping, Pouponière, CS Kpassèton, Clinique Beaux Bébés, Dispensaire St Camille de Sègbanou sont praticables à cause de leurs situations géographiques. Ces centres de santé sont en fait situés au bord ou non loin des voies bitumées. Cette impraticabilité des voies rend difficile l'accès aux soins de santé dans la zone sanitaire OKT. La photo 1 illustre l'état de la voie menant vers l'hôpital de zone qui est le premier centre de référence de la zone sanitaire OKT.

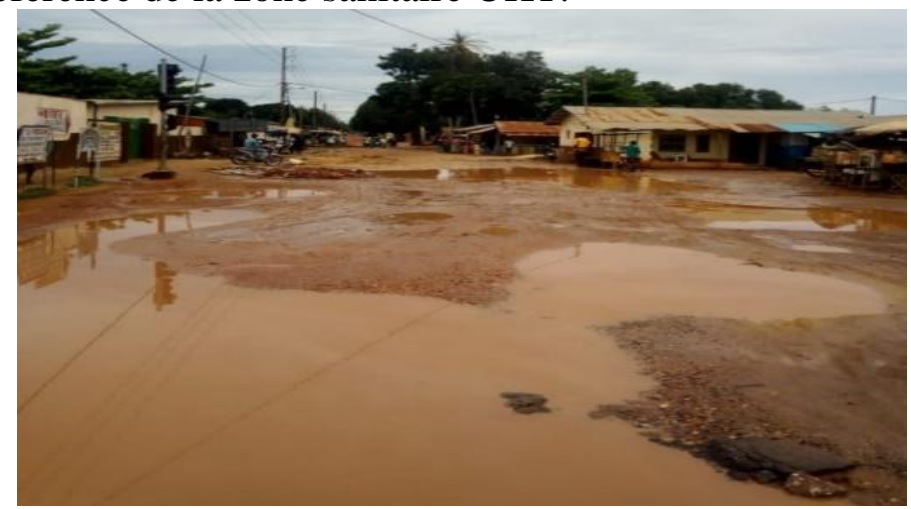

Photo 1 : Voie menant à l'Hôpital de zone OKT impraticable (Carrefour Gbèna dans l'arrondissement de Ouidah 2)

Prise de vue: TCHINKOUN, juin 2021

La photo 1 montre une vue partielle de la voie menant à l'hôpital de zone OKT. La voie d'accès à l'hôpital de zone n'étant pas bitumée, elle présente tout le long de l'année un état de dégradation. Elle est parfois rechargée par la mairie mais ce reprofilage ne règle que temporairement et à court terme le problème qui malheureusement demeure à moyen terme. Elle est très souvent impraticable surtout en saison pluvieuse. Ceci pose un problème crucial pour l'accessibilité aux soins de santé étant donné que cet hôpital est le seul Hôpital de référence de la zone sanitaire. 


\subsection{Capacité financière des enquêtés dans la zone sanitaire OKT}

Accéder aux services de santé, suppose que l'on ait les ressources financières nécessaires pour faire face aux coûts des différents actes. Le revenu du ménage, sa taille, c'est à dire le nombre de personnes à charge du chef de ménage influence l'itinéraire de soins de ceux qui ont des contraintes budgétaires selon Djigbenoudé cité par (TCHAOU, 2013 p. 23). L'indice de confort matériel a donc été calculé afin d'apprécier le niveau de vie et le confort des populations enquêtées en relation avec leur prise de décision. Le tableau 4 présente le récapitulatif du confort matériel des enquêtés.

Tableau IV : Confort matériel des enquêtés

\begin{tabular}{|l|l|l|}
\cline { 2 - 3 } \multicolumn{1}{c|}{} & Effectifs & $\%$ \\
\hline confort faible & 270 & 30 \\
\hline confort moyen & 450 & 50 \\
\hline confort élevé & 180 & 20 \\
\hline Total & 900 & 100 \\
\hline
\end{tabular}

L'analyse du tableau IV montre que $30 \%$ des enquêtés vivent dans un confort faible, $50 \%$ dans un confort moyen et $20 \%$ dans un confort élevé. On en déduit donc que les populations de la zone OKT ont un niveau de vie plutôt acceptable. Le niveau de vie étant l'un des facteurs majeurs qui empêchent les populations d'utiliser les services de soins de santé moderne, une corrélation a été faite pour apprécier la relation entre le niveau de vie du ménage et l'utilisation des types de centres de santé modernes.

\section{Analyse de la relation entre l'ICM et l'utilisation des centres de santé modernes}

Pour déterminer l'utilisation des différents types de centres de santé, une analyse factorielle des correspondances simple (AFC) a été effectuée sur le confort matériel des enquêtés. Les résultats de cette analyse révèlent que le premier axe explique à lui seul $87.47 \%$ des informations obtenues (figure 6), ce qui est largement suffisant pour faire une bonne synthèse des informations. 


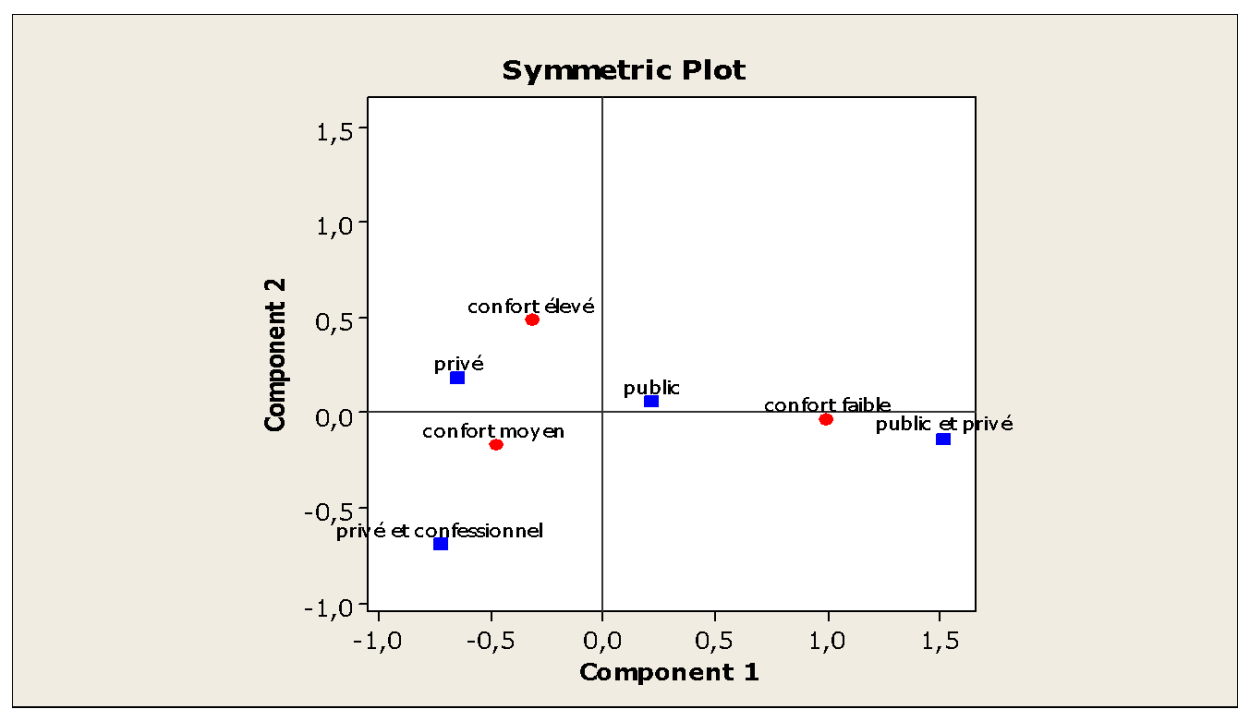

Figure 6 : Représentation des variables sur les plans factoriels 1 et 2 Source : Analyse des résultats, juin 2020

L'analyse factorielle retient deux axes factoriels (figure 6). A l'axe factoriel 1 sont corrélées toutes les variables sauf la variable confort élevé qui est corrélée à l'axe 2. Les variables "'confort faible", 'CS public", 'CS public et privé" sont corrélées positivement tandis que les variables "confort moyen", "CS privé" et "CS privé et confessionnel" sont corrélées négativement. La variable "'confort élevé" est corrélée quant à elle positivement à l'axe factoriel 2 . On en déduit que les populations utilisent tous les types de centres de santé quel que soit le confort. Mais les centres de santé publics sont plus utilisés par les populations vivant dans un confort faible faute de moyens financiers. Le coût des prestations pourrait donc être l'une des raisons qui amènent les populations à fréquenter les centres de santé publics.

\section{Analyse de la relation entre l'ICM et le recours à l'automédication}

Tableau V : Corrélation entre l'ICM et le recours à l'automédication

\begin{tabular}{|l|l|}
\hline ICM & $\begin{array}{l}\text { Proportion des enquêtés ayant recourt à } \\
\text { l'automédication en fonction du confort }\end{array}$ \\
\hline confort faible & 72.22 \\
\hline confort moyen & 80 \\
\hline confort élevé & 10 \\
\hline Chi2 = 29 $* * * \quad$ V de Cramer $=\mathbf{0 , 4 9 2} * * *$ \\
\hline Test significatif à 1\% $(* * *)$, à $5 \%(* *)$, Non significatif $(\mathrm{ns})$ \\
\hline \multicolumn{2}{|c|}{ Source : Analyse des résultats, juin 2020 }
\end{tabular}

L'analyse du tableau 5 montre une corrélation modérée (V de Cramer $=0,492)$ entre le confort matériel du ménage et le recours à l'automédication. 
On retient que, ce sont les ménages qui vivent dans des conforts faibles ou moyens c'est-à-dire qui ont des contraintes budgétaires qui s'adonnent plus à l'automédication. Les $10 \%$ d'enquêtés vivant dans un confort élevé et ayant recours à l'automédication montrent que les populations s'adonnent à l'automédication indépendamment du confort dans lequel ils vivent. Le manque de temps, la récurrence des épisodes morbides et la facilité d'acquisition de médicaments pour des symptômes d'une affection déjà traitée sont entre autres des facteurs prédisposant les populations à adopter, en première instance l'automédication. Le recours à l'automédication ne dépend donc pas du confort dans lequel vit le ménage. Notons que le paiement des coûts de services et soins de santé et des médicaments dépend fortement du niveau de vie du ménage.

L'évaluation de l'accès aux soins de santé montre que le système de santé du milieu d'étude est défaillant. De fait, l'offre de soins est très peu respectueuse des normes de l'OMS. Cette situation trouve son fondement dans la croissance incontrôlée de la population qui se fait ressentir sur d'autres facteurs à savoir le déficit d'infrastructures sanitaires, la carence en ressources humaines. D'autres facteurs que sont l'impraticabilité des voies d'accès aux centres de santé, l'incapacité financière d'une certaine tranche de la population à souffrir des soins de qualité menacent également le système de santé dans le milieu d'étude

\section{Discussion}

Les politiques sanitaires actuelles n'arrivent pas encore à suivre la croissance de la population. Pour preuve, la zone sanitaire compte environ 331.697 habitants mais reste toujours avec une seule zone sanitaire alors que la réorganisation du système sanitaire béninois intervenu en 1997, prévoit une zone sanitaire pour une population allant de 100.000 à 200.000 habitants. Aussi, peu d'infrastructures disponibles sont conformes aux normes de l'OMS surtout dans la Commune de Ouidah où les centres de santé sont non seulement pour la plupart vétustes, mais ne disposent pas non plus de logement pour le personnel soignant. L'absence de centre de santé dans certains arrondissements comme celui de Ouidah IV rend les populations plus vulnérables à toutes sortes de maladies. Ces résultats concordent avec ceux de Zondji (2019) qui selon les résultats de ses recherches sur la dynamique démographique et l'accès aux soins de santé modernes dans la zone sanitaire Allada-Toffo-Zè affirme que malgré les efforts fournis par l'Etat pour améliorer l'accès aux soins dans les hameaux du pays, l'offre publique de services de santé est encore insuffisante, au regard de la taille de la population. Abondant dans le même sens, Lerberghe et ses paires (1997) affirment que le premier défi de bon nombre de pays en développement reste la mise en place effective d'un système de santé de base. Dans beaucoup de pays, la couverture 
sanitaire des populations rurales reste incomplète. En termes d'infrastructures, les systèmes de santé sont souvent en retard sur une croissance démographique soutenue.

Le nombre de cas de la plupart des pathologies augmente au fil des années avec l'évolution démographique ce qui induit une augmentation des besoins en personnel de santé. Aucun des ratios médecin / habitants, IDE / habitants et SFE / habitants ne respecte les normes de l'OMS (1 Médecin pour 10000 habitants, 1 IDE pour 5000 habitants et 1 SFE pour 5000 habitants) dans la zone sanitaire. Alors qu'en dessous de ces différents seuils, la qualité des soins peut se dégrader en termes d'accueil des patients, de leur suivi et de la célérité dans les prestations. Certains centres de santé ne disposent pas d'infirmiers diplômés d'Etat et de sages-femmes et tout repose souvent sur les aides-soignantes et/ou infirmières brevetées qui ne sont pas habiletés à administrer des soins. Pour les communes de la zone sanitaire, on note un taux de couverture de $8.82 \%$ de médecin, $30.43 \%$ d'infirmiers d'Etat et $30.88 \%$ de sages-femmes d'Etat. Cette insuffisance quantitative en personnel de santé affecte négativement la qualité des soins offerts aux populations de la zone OKT. Ces résultats confirment ceux obtenus par Zambo (2012) et Tchaou (2013) sur les facteurs associés aux recours aux soins de santé primaires dans les formations sanitaires. Marchal (2003) vient confirmer que l'insuffisance d'un personnel qualifié est une autre contrainte majeure de l'offre de soins. Formé en nombre insuffisant, ce dernier est également mal distribué. Partout, les zones rurales restent mal couvertes.

La question de l'accès se pose également en termes de disponibilité de l'offre de soins. Le maillage géographique des centres de santé publics montre que la couverture sanitaire de la zone OKT est dense avec le rayon de $5 \mathrm{~km}$ et donc les formations sanitaires publiques peuvent être ralliées facilement par les populations. Toutefois quelques déserts sanitaires ou espaces de ségrégation sanitaire sont observés. Aussi, les pistes et voies non bitumées sont pour la plupart très dégradées dans la zone sanitaire du fait surtout de l'inondation alors qu'elles sont les plus pratiquées par la population pour aller dans leur centre de santé. Les résultats obtenus confirment les conclusions de l'OMS en 1995 qui stipulent que : la distance constitue un facteur très déterminant dans l'accès aux centres de santé. L'accessibilité physique et l'effet de distance sont de ce fait des variables indépendantes importantes dans le choix du type de recours aux services, aux soins de santé modernes. Ces résultats concordent également avec ceux de Franckel (2004) qui montre que l'éloignement par rapport aux structures sanitaires a une incidence majeure sur les pratiques thérapeutiques : la propension à consulter en structure sanitaire diminue avec la distance et, au contraire, la pratique d'automédication augmente. 
Accéder aux services de santé, suppose que l'on ait les ressources financières nécessaires pour faire face aux coûts des différents actes. Les résultats sociologiques montrent que le confort et la taille du ménage influence l'itinéraire de soins surtout de ceux qui ont des contraintes budgétaires. Ces résultats concordent avec ceux de Franckel (2004) qui montre que les pratiques thérapeutiques des différents ménages varient en fonction du statut économique et social de la cellule familiale : les cellules familiales de grande taille, qui jouissent d'une position avantageuse, consultent plus en structure sanitaire, alors que la précarité économique des ménages comptant un petit nombre d'enfants explique leur moindre propension à consulter en structure sanitaire.

\section{Conclusion}

La présente étude expose les contraintes liées à l'accès aux soins de santé face à l'explosion démographique de la zone sanitaire Ouidah/Kpomassè/Tori-Bossito. Au terme de cette étude, l'on retient que le sous-système de santé de la zone sanitaire $\mathrm{OKT}$, s'il s'insère dans le système national de santé ne répond plus aux normes démographiques actuelles et présente des dysfonctionnements qui l'empêchent de répondre à la demande d'une population croissante. Il y a donc urgence pour les autorités du secteur de la santé d'accompagner véritablement la croissance de la population en mettant à sa disposition des établissements hospitaliers et un nombre suffisant de professionnels de santé à la mesure de l'évolution démographique de ladite population. La construction des voies et la mise en œuvre des projets de mutuel de santé par l'Etat aideront à corriger les insuffisances de l'offre de soins dans la zone OKT.

\section{References:}

1. BONI, Gratien. 2014. Croissance démographique : implications socio-spatiales, environnementales et sanitaires dans la commune d'Abomey-calavi au Bénin. UAC/Bénin. Abomey-Calavi : EDP/FLASHS, 2014. p. 251, Thèse de Doctorat.

2. Bureau de zone OKT. 2020. Statistiques sanitaires. 2020. p. 72.

3. FRANCKEL, Aurélien. 2004. Les comportements de recours aux soins en milieu rural au Sénégal: le cas des enfants fébriles à Niakhar. Ecole doctorale Economie, Organisation et Société, UNIVERSITE PARIS X - NANTERRE. Centre de Recherche Populations et Sociétés - CERPOS, 2004. p. 465, Thèse de Doctorat.

4. INSAE. 2013. Recensement Général de la Population et de l'Habitat (2012): Synthèse des résultats . Cotonou.

5. Lerberghe, Wim Van, DE BROUWERE, Vincent et De Béthune, X. 1997. Hospitals in sub-Saharan Africa: why we need more of what does 
not work as it should, Tropical Medicine and International Health, 1997. pp. 799-808, Article. volume 2 no 8 .

6. Marchal, Bruno et Kegels, Guy. 2003. Health workforce imbalances in times ofglobalization: brain drain or professionalmobility? Department of Public Health, Institute of Tropical Medicine, Antwerp, Belgium, International journal of health planning and management, 2003. pp. 89-101, Article. 18.

7. PNDS. 2009. Plan National de Développement Sanitaire 2009-2018. Benin,. 2009.

8. Richard, Jean Luc. 2001. Accès et recours aux soins de santé dans la sous-préfecture de Ouèssè. Bénin: Faculté des Lettres Humaines de l'Université de Neuchâtel, 2001. p. 1134, Thèse.

9. SANTANA, Angèle. 2016. Coopération décentralisée et amélioration de l'accès aux soins dans la commune d'Abomey-Calavi. MIRD/FASHS. Abomey-Calavi, p. 84, Mémoire de Master.

10. TCHAOU, Gabin. 2013. Dynamique de population et offres de services de soins de santé modernes dans la zone sanitaire Abomeycalavi/ Sô Ava. EDP/ FLASH/ UAC. 2013. p. 342, Thèse de doctorat unique de Géographie.

11. ZAMBO, Yannick. 2012. Croissance démographique et développement en Afrique subsaharienne, Université, 2012. p. 82, Mémoire Master 2 en Assurance et Gestion des risques.

12. ZONDJI, Ghislain. 2019. Dynamique démographique et offres de services modernes de soins dans la zone sanitaire Allada-Toffo-Zè au Bénin. EDP/UAC. Abomey-Calavi, 2019. p. 232, Thèse de Doctorat. 